\title{
Haematopoietic stem cells and endothelial progenitor cells in healthy men: effect of aging and training
}

Dick H. J. Thijssen, ${ }^{1}$ Joost B. Vos, ${ }^{2}$ Caroline Verseyden, ${ }^{2}$ Anton Jan van Zonneveld, ${ }^{2}$ Paul Smits, ${ }^{3}$ Fred C. G. J. Sweep, ${ }^{4}$ Maria T. E. Hopman ${ }^{1}$ and Hetty C. de Boer ${ }^{2}$

${ }^{1}$ Department of Physiology, Institute of Fundamental and Clinical Movement sciences, Radboud University Nijmegen Medical Centre, The Netherlands

${ }^{2}$ Department of Nephrology, Leiden University Medical Centre, The Netherlands

${ }^{3}$ Department of Pharmacology and Toxicology, Radboud University Nijmegen Medical Centre, The Netherlands

${ }^{4}$ Department of Chemical Endocrinology, Radboud University Nijmegen Medical Centre, The Netherlands

\section{Summary}

The number of hematopoietic stem cells (HSC) and endothelial progenitor cells (EPC) is thought to be a marker for neovascularization and vascular repair. Because physical inactivity and aging are risk factors for cardiovascular diseases, these factors may influence the numbers of HSCs and EPCs. Therefore, we examined baseline and exercise-induced levels of HSCs and EPCs in sedentary and trained young and older men. To study the role of aging in eight sedentary young (19-28 years) and eight sedentary older men (67-76 years), baseline and acute exercise-induced numbers of HSCs (CD34 ${ }^{+}$-cells) and EPCs (CD34 ${ }^{+} /$VEGFR-2 ${ }^{+}$-cells) were quantified by fluorescenceactivated cell sorter (FACS) analysis. To examine the effect of chronic training, eight age-matched trained young men (18-28 years) were compared with sedentary young men, whereas older men performed an 8-week endurance training. Older men showed significantly lower baseline and exercise-induced levels of HSCs/EPCs than the young men $(P<0.05)$. In young and older men, acute exercise significantly increased HSCs $(P<0.01)$, but not EPCs. The absolute increase in numbers of HSCs was attenuated in older men $(P=0.03)$. Apart from the lower baseline numbers of EPCs after chronic training in older men, training status did not alter baseline or exercise-induced levels of HSCs/EPCs in young and older men. We concluded that advancing age results in lower circulating numbers of HSCs and EPCs and attenuates the acute exercise-induced

\section{Correspondence}

Dick H. J. Thijssen, MSc, Department of Physiology, Radboud University

Nijmegen Medical Centre, Geert Grooteplein-Noord 21, 6525 EZ, Nijmegen, The Netherlands. Tel.: 0031-243613650; fax: 0031 243540535;

e-mail: d.thijssen@fysiol.umcn.nl

Accepted for publication18 August 2006 increase in HSCs. Interestingly, in young as well as in older men chronic endurance training does not affect baseline and exercise-induced numbers of HSCs and EPCs.

Key words: aging; endothelial progenitor cell; endurance training; haematopoietic stem cell; single exercise bout.

\section{Introduction}

Recent evidence suggests that vascular morphology and function can be modulated by bone-marrow derived haematopoietic stem cells (HSC, CD34 $4^{+}$and endothelial progenitor cells (EPC, CD34 $^{+}$/VEGFR-2 ${ }^{+}$) (Asahara et al., 1997). In preclinical studies, it was shown that HSCS and EPCS can enhance angiogenesis, promote vascular repair, improve endothelial function, and induce neovascularization (Orlic et al., 2001; Hill et al., 2003; Rauscher et al., 2003; Urbich \& Dimmeler, 2004). HSCs and EPCs may play a role in the vascular responses and adaptations to exercise. A single exercise bout acutely mobilises circulating HSCs in healthy men (Laufs et al., 2005; Morici et al., 2005) and in subjects with cardiovascular disease (Adams et al., 2004; Laufs et al., 2004; Rehman et al., 2004; Steiner et al., 2005). Based on the fact that advancing age is associated with an impaired vascular function (Celermajer et al., 1994; DeSouza et al., 2000), the acute exercise-induced mobilization of HSCs may be influenced by age. Heiss et al. (2005) recently reported that advancing age is associated with a functional deficit of stem cells (such as proliferation, survival, and migration). The primary aim of the present study is to examine the effect of advanced age on the numbers of HSCs and EPCs in young and older men before ('baseline') and after ('acute exercise-induced') a single exercise bout (Fig. 1). We hypothesize that advancing age reduces baseline and acute exercise-induced levels of HSCS and EPCS.

Increased levels of HSCS and EPCS are associated with an enhanced endothelial function (Hill et al., 2003) and a decrease in cardiovascular risk (Hill et al., 2003; Werner et al., 2005). Interestingly, endurance training is associated with an improved endothelial function (Green et al., 2004) and a decrease in cardiovascular risk (Paffenbarger et al., 1986) as well. As such, one may speculate about a possible influence of the training status (i.e. trained vs. untrained) on the baseline or exercise-induced numbers of HSCS and EPCS. Therefore, the second aim of the study is to examine baseline and exercise-induced numbers of HSCS and EPCs in an age-matched population of sedentary and endurance trained young men. We extended our observations on the possible link between training status and numbers of HSCS and EPCS by training the older men for 8 weeks (Fig. 1). We hypothesize that a higher-training status leads to an elevation in baseline and exercise-induced numbers of HSCs and EPCS in young as well as in older men. 
Table 1 Group characteristics of the sedentary $(n=8)$ and trained $(n=8)$ young men and the older men $(n=8)$ before (sedentary) and after (trained) the 8-week exercise training (mean $\pm \mathrm{SE}$ ). (HDL, high-density lipoprotein; LDL, low-density lipoprotein; $\mathrm{VO}_{2 \text { max }}$, maximal oxygen consumption; bpm, beats per minute; RER, respiratory exchange ratio; HSC, haematopoietic stem cell; EPC, endothelial progenitor cell)

\begin{tabular}{|c|c|c|c|c|}
\hline & \multicolumn{2}{|l|}{ Young men } & \multicolumn{2}{|c|}{ Older men $(n=8)$} \\
\hline & $\begin{array}{l}\text { Sedentary } \\
(n=8)\end{array}$ & $\begin{array}{l}\text { Trained } \\
(n=8)\end{array}$ & Sedentary & Trained \\
\hline Activity, h/week & $0.8 \pm 0.9$ & $10.4 \pm 2.6^{*}$ & $0.5 \pm 0.8$ & $2.5 \pm 0.8^{*}$ \\
\hline Body mass index & $22.4 \pm 1.7$ & $22.8 \pm 1.2$ & $25.4 \pm 2.6 \dagger$ & $25.2 \pm 2.6$ \\
\hline Cholesterol, $\mathrm{mmol} \mathrm{L}^{-1}$ & $4.4 \pm 1.4$ & $4.2 \pm 0.8$ & $4.8 \pm 0.4$ & $4.6 \pm 0.1$ \\
\hline Triglycerides, mmol L $\mathrm{L}^{-1}$ & $1.5 \pm 0.8$ & $0.9 \pm 0.4$ & $1.0 \pm 0.3$ & $1.0 \pm 0.2$ \\
\hline $\mathrm{HDL}, \mathrm{mmol} \mathrm{L}^{-1}$ & $1.2 \pm 0.3$ & $1.6 \pm 0.2^{*}$ & $1.2 \pm 0.2$ & $1.2 \pm 0.1$ \\
\hline $\mathrm{LDL}, \mathrm{mmol} \mathrm{L}^{-1}$ & $2.5 \pm 1.1$ & $2.2 \pm 0.7$ & $3.2 \pm 0.3$ & $3.0 \pm 0.1$ \\
\hline \multicolumn{5}{|l|}{ Incremental exercise test } \\
\hline HR rest, bpm & $62 \pm 6$ & $54 \pm 7^{*}$ & $60 \pm 4$ & $52 \pm 3^{*}$ \\
\hline HR max, bpm & $194 \pm 11$ & $195 \pm 8$ & $165 \pm 7$ & $166 \pm 8$ \\
\hline $\mathrm{VO}_{2 \max }, \mathrm{mL} / \mathrm{kg} \mathrm{\operatorname {min } ^ { - 1 }}$ & $49.0 \pm 4.0$ & $58.5 \pm 7.5^{\star}$ & $30.8 \pm 4.8+$ & $33.3 \pm 5.5$ \\
\hline Workload max, W & $341 \pm 41$ & $393 \pm 53^{*}$ & $171 \pm 32+$ & $201 \pm 39 *$ \\
\hline Lactate max, mmol mL $\mathrm{m}^{-1}$ & $9.1 \pm 1.6$ & $12.0 \pm 1.9^{*}$ & $7.7 \pm 2.1$ & $7.6 \pm 1.2$ \\
\hline RER & $1.24 \pm 0.04$ & $1.19 \pm 0.04^{*}$ & $1.20 \pm 0.13$ & $1.17 \pm 0.06$ \\
\hline \multicolumn{5}{|l|}{ Resting values stem cells } \\
\hline HSC, cells $\mathrm{mL}^{-1}$ & $412 \pm 70$ & $355 \pm 95$ & $119 \pm 21 \dagger$ & $95 \pm 17$ \\
\hline $\mathrm{EPC}$, cells $\mathrm{mL}^{-1}$ & $154 \pm 43$ & $185 \pm 96$ & $35 \pm 12+$ & $19 \pm 8^{*}$ \\
\hline
\end{tabular}

$* P<0.05$ (sedentary vs. trained).

$+P<0.05$ (sedentary young vs. sedentary older men).

\section{Results}

\section{Effect of aging on HSC and EPC in sedentary healthy men}

Maximal oxygen consumption in sedentary young men was significantly higher than in older men, while body-mass index was significantly lower in young than in older men (Table 1). Lipid profile was not different between both groups.

\section{Baseline}

Numbers of HSCs and EPCS in young men were significantly higher than in older men ( $t$-test, $P<0.05$, Table 1). Plasma vascular endothelial growth factor (VEGF) concentration in young men was significantly lower than in older men (Table 2).

Single exercise bout

The single exercise bout in sedentary young and older men significantly increased the numbers of HSCs (Fig. 2A). Numbers of EPCs in young and older men did not change following an acute exercise bout (Fig. 2B). Plasma levels of VEGF in young men were significantly increased by cycling, while older men showed no change (Table 2).

\section{Effect of training status on HSC and EPC in young men}

Maximal oxygen consumption, maximal workload, blood lactate production, and $\mathrm{HDL}$ in the trained young men were significantly
Table 2 Plasma concentrations VEGF (in pg $\left.\mathrm{mL}^{-1}\right)$ in sedentary $(n=8)$ and trained $(n=8)$ young men and the older men $(n=8)$ before (sedentary) and after training (trained). P-value represents a Student's $t$-test (in young men) or a Mann-Whitney $U$-test (in older men) to assess differences between baseline and exercise-induced (post-EX). Values are means (lowest value-highest value) for the young men and median (lowest value-highest value) for the older men

\begin{tabular}{lcr}
\hline & Baseline & Post-EX \\
\hline $\begin{array}{l}\text { Young men }(n=8) \\
\quad \text { Sedentary }\end{array}$ & $255(209-359)$ & $395(275-754) \dagger$ \\
$\quad$ Trained & $369(169-723)$ & $394(218-551)$ \\
Older men $(n=7)$ & & \\
$\quad$ Sedentary & $1239(488-20316)^{*}$ & $1429(591-20872)$ \\
$\quad$ Trained & $569(172-18205)^{*}$ & $590(244-12561)$ \\
\hline
\end{tabular}

${ }^{\star} P<0.05$ vs. young men.

$+P<0.05$ baseline vs. postexercise.

higher than in the sedentary young men. Resting heart rate and the respiratory exchange ratio (RER) were significantly lower in trained than in sedentary young men. Other characteristics did not differ between both groups (Table 1).

Baseline

Circulating numbers of HSCs and EPCs (Table 1), and VEGF plasma levels (Table 2) at baseline were similar between sedentary and trained young men.

\section{Single exercise bout}

The acute exercise-induced increase in numbers of HSCs in sedentary and trained young men was not influenced by chronic training (Fig. 3A). Numbers of EPCs in sedentary and trained young men did not change following the single exercise bout (Fig. 3C). Plasma levels of VEGF in trained young men did not change by the acute exercise bout (Table 2).

\section{Effect of training on HSC and EPC in older men}

The 8-week endurance training significantly decreased resting heart rate and increased maximal workload (Table 1) in the older men. Maximal oxygen consumption was increased, although not significantly ( $t$-test, $P=0.09)$.

Baseline

Baseline numbers of HSCs were not altered after endurance training (Table 1), while EPCs (Table 1) and plasma levels of VEGF (Table 2) were significantly lower after training.

Single exercise bout

The acute exercise-induced increase in numbers of HSCs in older men is independent of physical fitness (Fig. 3B). Before as well as after the endurance training, the single exercise bout test did not change numbers of EPCS (Fig. 3D) and VEGF concentrations (Table 2). 
Fig. 1 Schematic presentation of the experimental protocol. After a 5-min seated rest venous blood was taken. Subsequently, 10 min after cessation of the cycling test, a second venous sample was taken. The sedentary and trained young men performed this protocol once, while the sedentary old men completed the protocol before and after the 8-week endurance training.

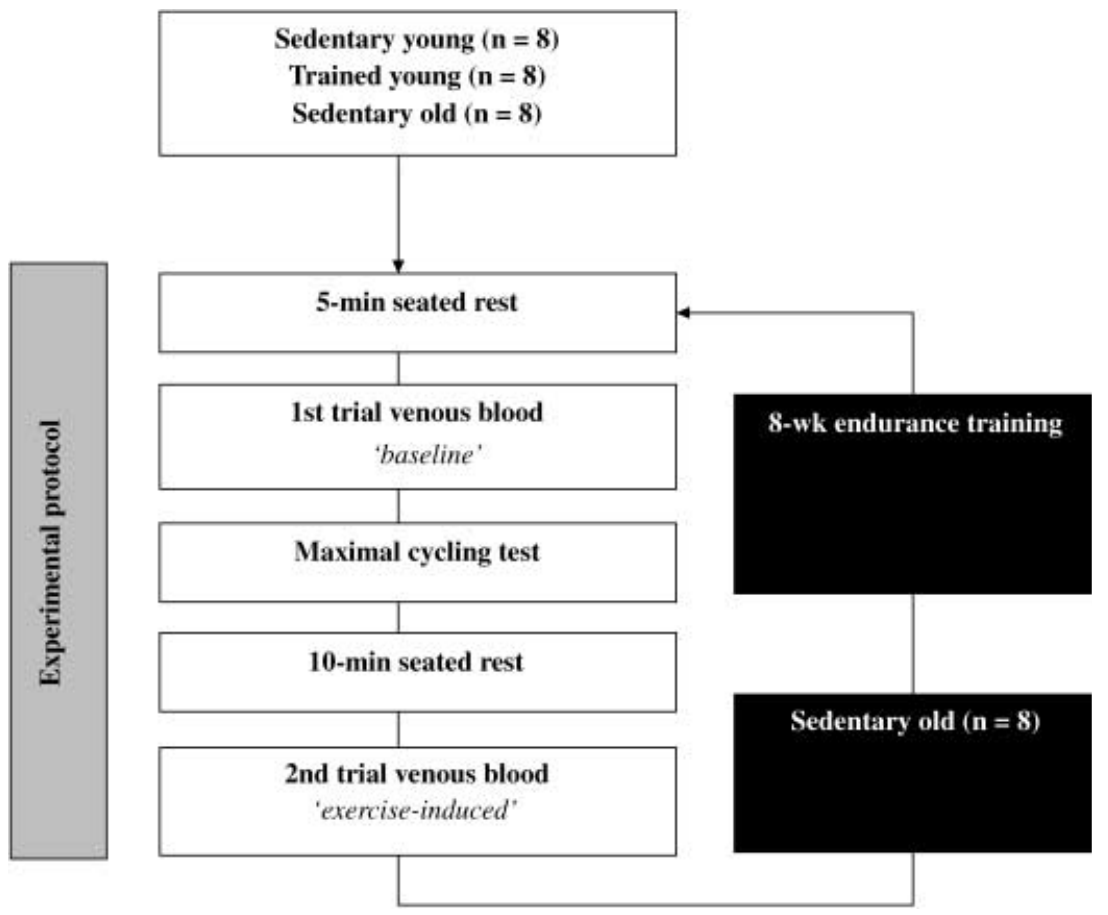

A

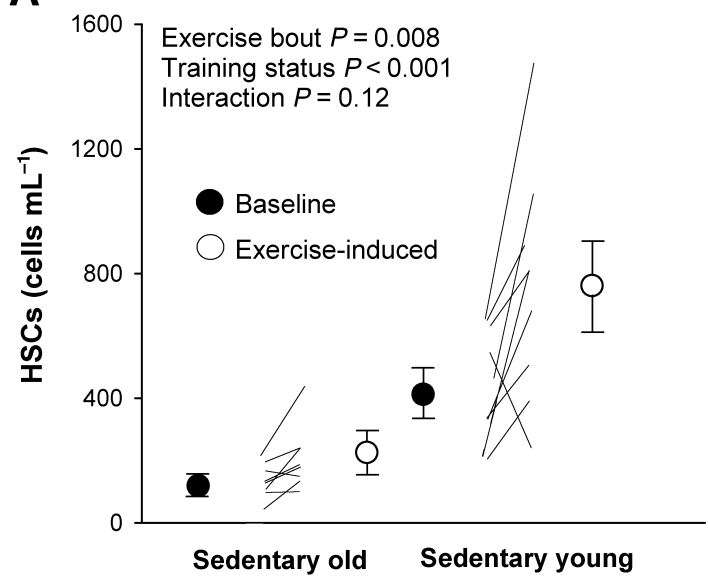

B

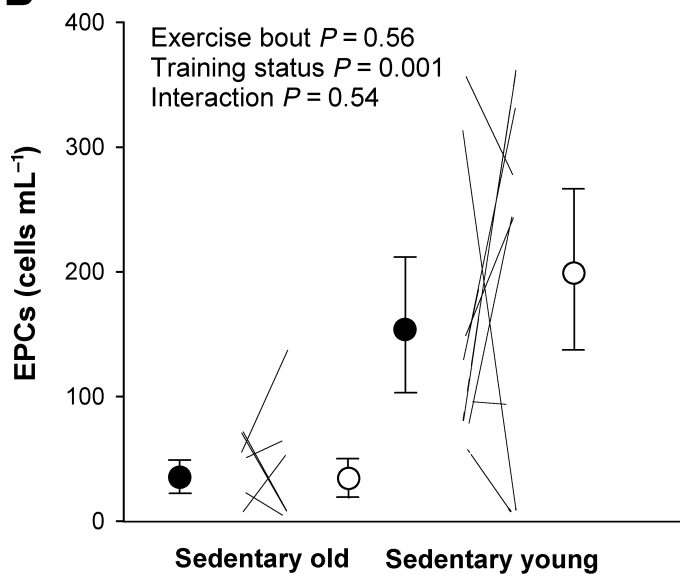

Fig. 2 Individual and average baseline (solid circles) and exercise-induced levels (open circles) of circulating numbers of haematopoietic stem cells (HSCs, A) and endothelial progenitor cells (EPCS, B) in sedentary young and older men (both $n=8$ ). Average values represent means \pm SE. The two-way analysis of variance (ANOVA) indicates higher baseline and exercise-induced numbers of HSCs in young men compared with older men, while the exercise-induced increase in HSCs showed an age-dependent tendency.

\section{Linear regression analysis}

The linear regression analysis indicated a significant relationship between baseline and exercise-induced numbers of HSCS (Fig. 4A, $P=0.001$ ) and EPCs (Fig. 4B, $P<0.001$ ) in both sedentary and trained healthy men.

The absolute acute exercise-induced increase in numbers of HSCs in young men (due to the higher baseline numbers of HSCs) was larger than in older men (Fig. 5A). However, the relative acute exercise-induced change in HSCs was similar between young and older men (Fig. 5B). As such, the relative exercise-induced increase in HSCS is independent of age.
The exercise-induced relative increase in numbers of HSCs and EPCs in young $\left(r^{2}=0.07\right.$ and $0.001, P=0.31$ and 0.93 , respectively) as well as in older men $\left(r^{2}=0.12\right.$ and $0.04, P=0.20$ and 0.63 , respectively) did not correlate with the relative change in VEGF plasma levels. Maximal oxygen consumption did not correlate with the baseline or exercise-induced levels of HSCs $\left(r^{2}=0.17\right.$ and $0.07, P=0.12$ and 0.33 , respectively) nor EPCS in young men $\left(r^{2}=0.10\right.$ and $0.20, P=0.23$ and 0.08 , respectively). Also older men show no correlation between maximal oxygen consumption and baseline or exercise-induced levels of $\mathrm{HSCS}\left(r^{2}=0.001\right.$ and $0.03, P=0.90$ and 0.56 , respectively) nor EPCS $\left(r^{2}=0.05\right.$ and $0.07 ; P=0.0 .42$ and 0.51 , respectively). 
A
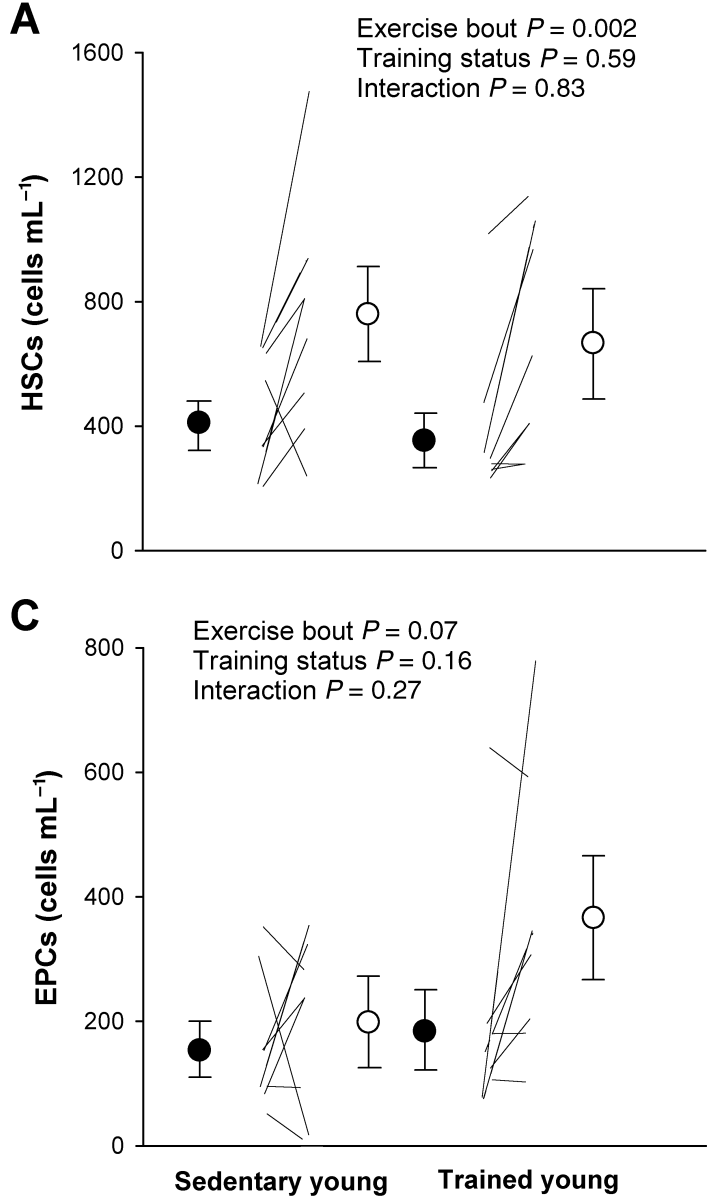

B

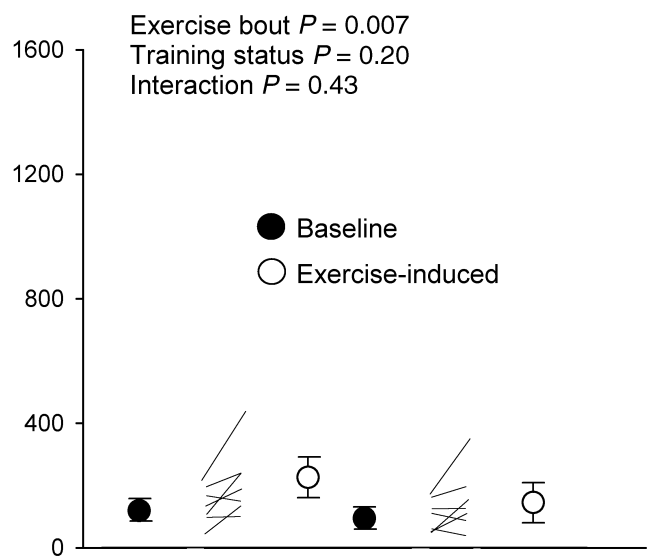

D

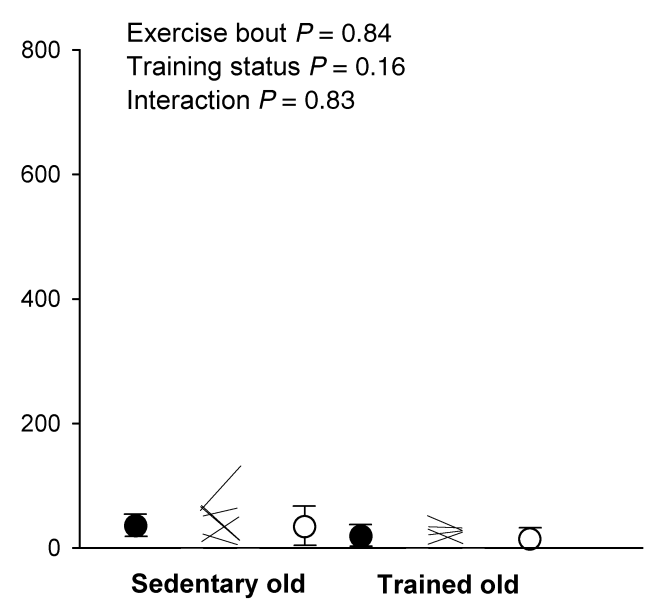

Fig. 3 Baseline (filled circles) and exercise-induced levels (open circles) of circulating numbers of haematopoietic stem cells (HSC, A-B) and endothelial progenitor cells (EPC, C-D). In young men, numbers of HSCs (A) and EPCS (C) were presented in the sedentary $(n=8)$ and trained $(n=8)$ subpopulation. In older men $(n=8)$, numbers of HSCS (B) and EPCS (D) were represented before and after training. Values represent mean \pm SE. Baseline and exercise-induced numbers of HSCs were independent of physical fitness. Also the baseline and exercise-induced numbers of EPCs were independent of physical fitness.
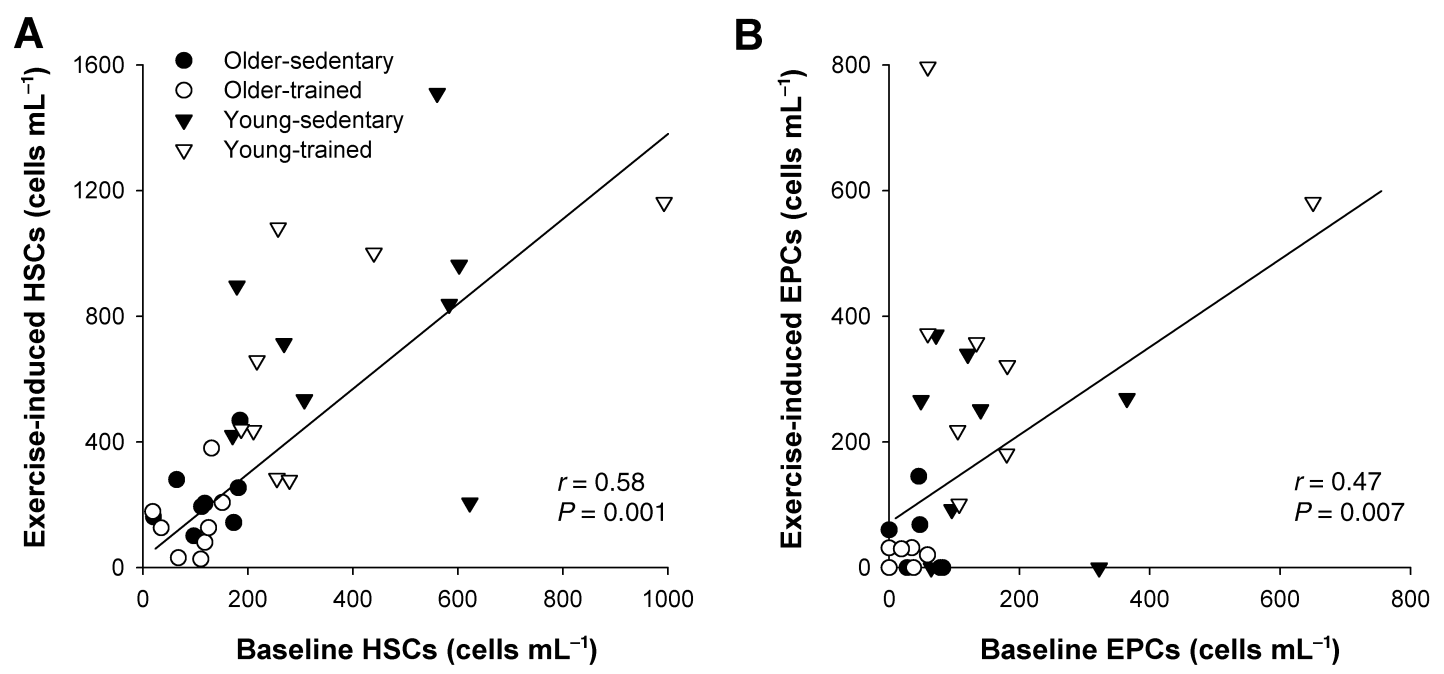

Fig. 4 Linear regression between baseline and exercise-induced numbers of haematopoietic stem cells (HSC, A) and endothelial progenitor cells (EPC, B) in sedentary ( $n=8$, open triangles) and trained young men ( $n=8$, open circles) and older men ( $n=8$ ) before (solid triangles) and after training (solid circles). This analysis assessed the correlation between baseline and exercise-induced numbers of HSCS and EPCS. 

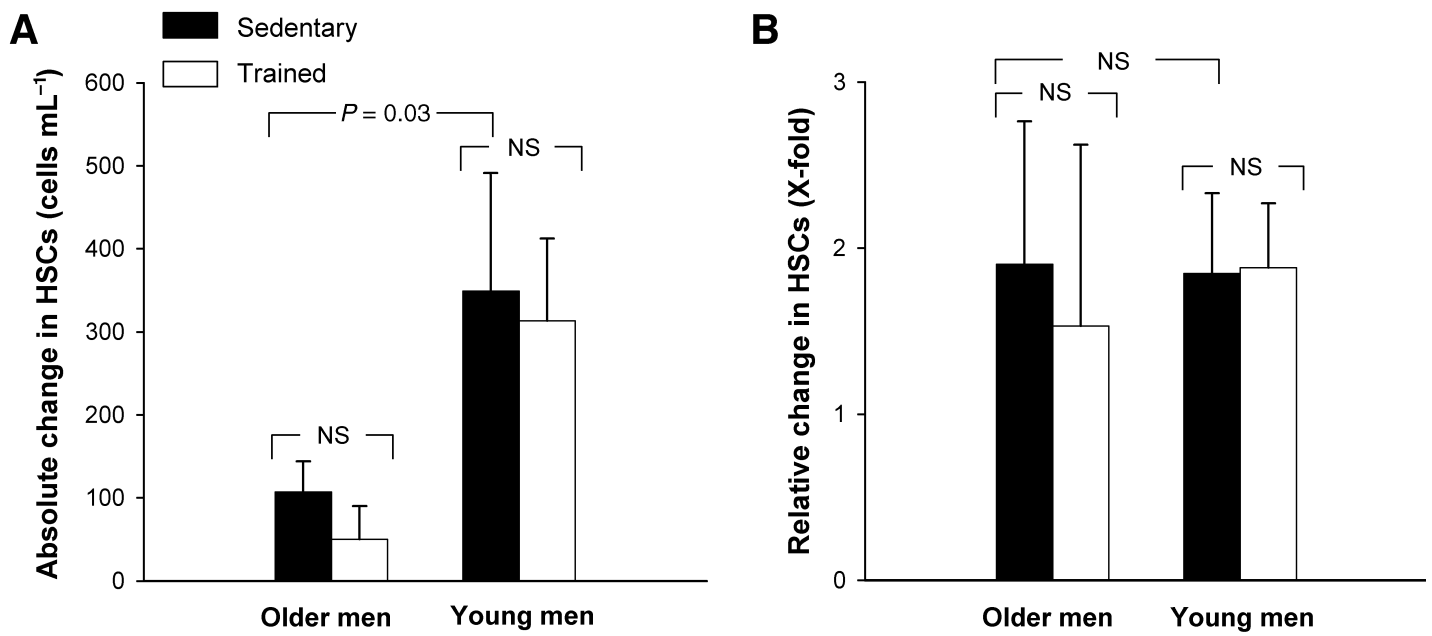

Fig. 5 Exercise-induced change in haematopoietic stem cells (HSC) in sedentary (black bars) and trained (white bars) young and older men, expressed as the absolute $\left(A\right.$, in cells $\mathrm{mL}^{-1}$ ) and relative ( $B$, in X-fold change) from baseline numbers. Because data were not normally distributed, a Mann-Whitney $U$-test (between groups) or Wilcoxon signed rank test (within groups) was used to assess differences between groups. Values are means \pm SE.

\section{Discussion}

This study provides several interesting findings. First, the number of HSCs and EPCs in young men is higher than in older men. Second, this inverse correlation in age and HSC numbers was not altered after endurance training in young men, whereas EPC numbers decreased further after 8 weeks of endurance training of older men. Third, a single exercise bout in young as well as in older men acutely mobilizes HSCs, but not EPCs, to the peripheral blood. Fourth, the single exercise bout-induced change in numbers of HSCs is not influenced by training status, while advanced age is associated with an attenuated mobilization in numbers of HSCs. Fifth, numbers of HSCs and EPCs were not correlated to VEGF levels. Thus, our results suggest an agerelated decrease in baseline and exercise-induced levels of HSCS and EPCs, which is not influenced by a change in training status.

We compared three well-defined populations, based on their age and training status. The maximal oxygen consumption of the sedentary (Miyachi et al., 2004) and active (Franzoni et al., 2004; Kraus et al., 2004; Laufs et al., 2005) young men and the older men (Tanaka et al., 2000; Monahan et al., 2001; Tanaka et al., 2002; Eskurza et al., 2004) is comparable with previous studies. Although the cycling training in older men did not significantly improve maximal oxygen consumption, seven out of eight subjects increased their maximal oxygen consumption and all men completed a higher maximal workload during the posttraining cycling test. Moreover, the $\sim 8 \%$ increase in oxygen consumption is larger than reported in previous studies in which older men were trained for 12 weeks (DeSouza et al., 2000; Tanaka et al., 2000; Tanaka et al., 2002).

\section{Effect of aging on numbers of HSCs and EPCs}

Sedentary older men demonstrate markedly lower baseline levels of HSCS and EPCs than their younger peers. Because
EPCs correlate inversely with the incidence of cardiovascular events (Werner et al., 2005), the age-related down-regulation in numbers of HSCS and EPCS may contribute to the increased cardiovascular risk in the older population. Although lower baseline numbers of HSCs and EPCs with advancing age is also reported in previous studies (Vasa et al., 2001; Schmidt-Lucke et al., 2005), others (Heiss et al., 2005; Chen et al., 2006; Shaffer et al., 2006) reported no difference between young and older men. This variation among studies likely results from differences in the method used. We analyzed HSCs and EPCs in whole blood, while for studies in which no difference was found (Heiss et al., 2005; Chen et al., 2006; Shaffer et al., 2006), numbers of stem cells was measured in the mononuclear cell fraction, isolated by Ficoll density gradient centrifugation. This method may cause loss of cells and underscores the importance of the choice of the method used.

\section{Effect of a single exercise bout on HSCs and EPCs}

The finding that acute exercise increases the level of HSCs is in accordance with a previous study of healthy young subjects (Morici et al., 2005). Given the ability of HSCs to promote angiogenesis and promote vascular repair (Orlic et al., 2001; Hill et al., 2003; Rauscher et al., 2003; Urbich \& Dimmeler, 2004), the acute exerciseinduced mobilization may serve as a physiological repair or adaptation mechanism. To examine whether the aging process influences the exercise-induced number of HSCs, we assessed this response in young and older men. Young men report a significantly larger increase in exercise-induced absolute numbers of HSCs than older men. However, expressing the increase as a relative change, our data demonstrate no differences between young and older men in the exercise-induced increase in HSCS. This indicates that the larger exercise-induced number of HSCS in young men is primarily caused by the higher baseline numbers of HSCs in young compared with older men. 
Acute exercise did not alter the numbers of EPCs in young and older sedentary men, which is consistent with a previous study using a similar exercise protocol for middle-aged healthy subjects and middle-aged patients suffering from nonischemic coronary artery disease (Adams et al., 2004). Interestingly, in ischemic coronary artery disease patients, numbers of EPCS were significantly increased, which coincided with higher levels of VEGF, implying that the ischemic stimulus is the initiating factor for EPC increase. During an ischemic episode, VEGF levels seem to correlate with EPC levels in patients with cardiovascular disease (Shintani et al., 2001; Adams et al., 2004) as well as in animal models (Asahara et al., 1997, 1999), while this was not the case for basic fibroblast growth factor (b-FGF), granulocytemacrophage colony stimulating factor (GM-CSF), and tumor necrosis factor alpha (TNF- $\alpha$ ). Interestingly, we and others (Rehman et al., 2004; Laufs et al., 2005) demonstrated that the exercise-induced increase in stem cells is not accompanied by a change in VEGF plasma levels in healthy men. In the absence of an ischemic stimulus VEGF levels may therefore not predict an exercise-induced change in HSCS and EPCS in healthy men. Although we can only speculate, the well-established exerciseinduced increase in nitric oxide bioavailability may be a potential mechanism by which mobilization of EPCs is regulated. This is supported by the finding that in mice the exercise-mediated upregulation of EPCs was absent in nitric oxide synthase 'knockout' mice or in the presence of a nitric oxide synthase inhibitor in healthy mice (Laufs et al., 2004). In addition, the hepatocyte growth factor, a potential stimulator of bone marrow derived cells (Ishizawa et al., 2004), may also be involved in the upregulation of HSCs. Recently, the hepatocyte growth factor was demonstrated as a good marker for progenitor proliferation at rest and after an acute exercise bout in healthy controls and in subjects with chronic pulmonary obstructive disease (Palange et al., 2006).

\section{Effect of training on numbers of HSCs and EPCs}

Interestingly, neither baseline numbers nor the exercise-induced numbers of HSCs and EPCs are different between sedentary and age-matched trained young men. This suggests that chronic endurance training in young men does not influence baseline or exercise-induced numbers of HSCS and EPCs. This is supported by the lack of correlation between oxygen consumption and levels of HSCS and EPCs in young men. To further extend this knowledge, we examined the older men after 8 weeks of training. Apart from a small but significant decrease in baseline EPCs levels, there are no differences in baseline and exerciseinduced levels of HSCs and EPC in older men after training. This supports our hypothesis that training status does not influence baseline or exercise-induced HSCs and EPCs.

To date, endurance training as an intervention to alter numbers of HSCs and EPCs is only examined in patients with peripheral arterial occlusive disease, coronary artery disease, or with cardiovascular risk factors (Laufs et al., 2004; Sandri et al., 2005; Steiner et al., 2005). Increased numbers of EPCs have been reported only when patients have symptomatic ischemia during exercise training (Sandri et al., 2005). Our finding of a decrease in numbers of EPCs in healthy older men after endurance training prompts us to question the physiological mechanism, although we can only speculate. It is noteworthy that our participants were free of cardiovascular risk factors, which is in marked contrast with the populations in previous studies (Laufs et al., 2004; Sandri et al., 2005; Steiner et al., 2005). In healthy older men, the EPCs may be involved in exercise-induced angiogenesis and/or repair processes, which consequently could result in lower baseline numbers of EPCs. On the other hand, bioavailability of nitric oxide is decreased during aging (Taddei et al., 2000), which may also affect the number of circulating EPCs. Interestingly, nitric oxide has been shown to differentially support mobilization of endothelial committed progenitors, but did not affect HSCs. Under nitric oxide-deficient conditions, granulocyte-colony stimulating factor (G-CSF) failed to increase EPC numbers, while the HSC population was unaffected (Ozuyaman et al., 2005). This may, at least in part, explain the decrease in EPCs upon chronic training in older subjects. Nevertheless, the responses of HSCS and EPCs to endurance training may differ between health and disease.

\section{Limitations}

In addition to the quantity (numbers), the quality of stem cells can also be examined (survival, migration, and proliferation). Advancing age, for example, has recently been associated with functional deficits of the stem cells (Heiss et al., 2005). As such, physical exercise may lead to changes in functional characteristics, rather than in the numbers of HSCs or EPCs. Future studies should examine changes in stem cell function after exercise training.

Comparing the results from the trained young men with the trained older men, one should take the amount of weekly exercise into account. The duration of training in older men is relatively short ( 8 weeks, three sessions per week), while young men trained chronically ( $>8 \mathrm{~h}$ a week). Despite these differences, the young as well as older trained men demonstrated baseline and acute exercise-induced values of HSCS and EPCS that are comparable to their sedentary counterparts.

In conclusion, this study demonstrates that advancing age is associated with lower numbers of circulating HSCS and EPCS, while the exercise-induced mobilization of HSCs is attenuated in older compared with young healthy men. Interestingly, in healthy young as well as in older men, endurance training does not affect baseline or exercise-induced numbers of HSCS or EPCs.

\section{Experimental procedures}

\section{Subjects}

Twenty-four participants, free of any cardiovascular disease and not on any medication known to interfere with the cardiovascular system, were recruited. Subjects had never smoked 
or stopped smoking at least 15 years ago. To examine the effect of aging, these healthy participants were parsed into two groups on the basis of age: eight older sedentary men (6776 years) and eight young sedentary men (19-28 years; Table 1). Sedentary was defined as less than $1 \mathrm{~h}$ of exercise per week for the past year or longer. To address the influence of the training status, the group of young sedentary men was compared with an age-matched population of endurance-trained young men $(n=8,18-28$ years, $\geq 8 \mathrm{~h}$ aerobic exercise per week). In addition, the older men performed an 8-week cycling training. Subjects underwent a physical examination, whereby subjects with abnormalities in a 12-lead electrocardiogram (ECG) at rest and/or an ankle-brachial pressure index of $<0.90$ were excluded. The study was approved by the hospital ethics committee and conformed with the principles outlined in the Declaration of Helsinki. All subjects gave their written informed consent before participation.

\section{Experimental design}

All participants performed a single maximal exercise bout on a cycling ergometer. Before and directly after the single exercise bout, blood was collected to analyze baseline and acute exercise-induced numbers of circulating HSCS and EPCS. The older men performed an 8-week endurance training. Subsequently, the same experimental protocol was repeated after the final training session (Fig. 1).

\section{Single exercise bout}

Experiments started between $08: 30$ and 10:00 hours. Subjects refrained from caffeine for at least $18 \mathrm{~h}$ and did not perform any strenuous activities at least $24 \mathrm{~h}$ before testing. Subjects were advised to have a light breakfast. All tests were performed in a temperature-controlled room $\left(20 \pm 1^{\circ} \mathrm{C}\right)$. Before the single exercise bout, subjects were seated in the upright position for at least $5 \mathrm{~min}$ before the baseline blood sample was drawn from an antecubital vein. The single exercise bout was performed on an electrically braked leg-cycling ergometer (Lode, Angio300, Groningen, The Netherlands), using a multistage protocol. Young men increased their workload by $20 \mathrm{~W}$ per minute, starting at $20 \mathrm{~W}$, until exhaustion, whereas older men used steps of $10 \mathrm{~W}$ per minute. Oxygen consumption was measured continuously in all groups using a gas-analyzer (Jaeger Benelux BV, Breda, The Netherlands). Maximal oxygen consumption $\left(\mathrm{VO}_{2 \max }\right)$ was analyzed as the mean of the last minute of the single exercise bout. In addition, blood lactate level (Roche Diagnostics GmbH, Mannheim, Germany) was measured before and 2 min after cessation of the test using capillary blood. Heart rate (HR) was recorded continuously. Subjects were verbally encouraged to continue for as long as possible. The criterion used to assess $\mathrm{VO}_{2 \max }$ included (1) HR in excess of $90 \%$ of age-predicted maximum (220-age); (2) RER $\geq 1.10$; and (3) identification of a plateau ( $\leq 150 \mathrm{~mL}$ increase) in $\mathrm{VO}_{2}$ despite a further increase in workload (Gavin et al., 2004). In all tests, at least two of three criteria were met. Finally, 10 min after cessation of the cycling test, the post-exercise blood sample was taken (Rehman et al., 2004).

\section{Measurements}

Enumeration of circulating HSCS and EPCS

For enumeration of circulating HSCs and EPCs, flow cytometric analysis was performed using a multiparametric gating strategy based on the International Society of Hematotherapy and Graft Engineering. This lyse/no wash method uses Trucount tubes (Becton Dickinson, Franklin Lakes, NJ, USA) that contain a defined number of brightly fluorescent microbeads, permitting the acquisition of absolute counts of cells, even at very low numbers. Circulating HSCs are defined as cells with low-expression of CD45, positive for CD34, and located in the lympho-gate on a side- and forward-scatter plot. This gating strategy was extended by calculating the number of $\mathrm{CD}_{3} 4^{+}$cells that also express vascular endothelial growth factor receptor-2 (VEGFR2) to define the number of EPCs. This strategy avoids inclusion of mature endothelial cells, which are also positive for CD34 and VEGFR-2, because they are located outside the lymphogate.

Within $2 \mathrm{~h}$ of blood-withdrawal, $50 \mu \mathrm{L}$ of EDTA-anticoagulated whole blood was added per Trucount tube (two per subject) by reverse pipetting and directly labeled antibodies were added: CD45-PerCP, CD34-FITC (BD Biosciences, Erembodegem, Belgium) and VEGFR-2-PE (R \& D Systems, Minneapolis, MN, USA). After 30 min incubation on ice and in the dark, cells were fixed using FACS-lysing solution (BD Biosciences) and the samples were measured within $24 \mathrm{~h}$ using a fluorescenceactivated cell sorter (FACS)-Calibur (BD Biosciences). A total of $500000 \mathrm{CD} 45^{+}$cells were measured (excluding the beads) and the number of HSCs and EPCs per milliliter blood was calculated.

\section{VEGF assay}

Plasma VEGF was measured, based on its suggested ability to mobilise EPCs into the blood (Asahara et al., 1999). Plasma VEGF levels were determined with a customized enzyme-linked immunosorbent assay (ELISA; Department of Chemical Endocrinology, Radboud University Nijmegen, The Netherlands). The assay measures $V_{E G F} 165$ and $V E G F_{121}$; the main isoforms of VEGF in blood. Details of the assay, including specificity and performance, have been described previously (Span et al., 2000).

\section{Endurance training}

The older men performed endurance training for 8 weeks (three sessions per week including cycling exercise) with at least 1 day between subsequent training sessions, under the supervision of a researcher. A cycling ergometer (Lode, Angio300) was used for endurance training. Each session started with a 10-min warming-up at $65 \%$ of the individual heart rate reserve (HRR). After muscle stretching, the exercise protocol was followed with 
20-min cycling exercise at $65 \%$ of the individual HRR. As their exercise tolerance improved throughout the training period, the intensity of the exercise bouts was increased by $5 \%$ HRR to a maximum of $85 \%$ of the HRR. In the first training session, the average workload was $157 \pm 48 \mathrm{~kJ}$ and in the twenty-fourth (i.e. last) training session, $234 \pm 50 \mathrm{~kJ}$. Adherence to the exercise prescription was documented through the use of HR monitors (s610, Polar, Brooklyn, NY, USA).

\section{Statistics}

The main goal of this study is to compare the exercise-induced change in levels of HSCs and EPCs. Based on the average exerciseinduced changes from previous studies (Laufs et al., 2004; Morici et al., 2005; Steiner et al., 2005) and an alpha of 0.05, we calculated that eight subjects per group would be necessary to achieve a power of $80 \%$. For the circulating numbers of HSCs and EPCs, a two-way mixed-plot factorial analysis of variance (single exercise bout $\times$ age or single exercise bout $\times$ training status) was used [Statistical Package for the Social Science (SPSS), version 12.0] (Ryan et al., 2006). Unpaired Student's t-tests were used to compare differences in all other variables between groups (young vs. old and trained vs. sedentary young men), while a paired Student's $t$-test was used to assess differences before and after endurance training in older men. Multiple linear regression was performed to identify relationships between variables. Results are expressed as mean \pm SE. When data are not distributed normally, data are presented as median [lowest value - highest value], and a nonparametric test was used to compare differences between (Mann-Whitney U-test) or within groups (Wilcoxon's signed rank test). A two-sided $P$-value of $\leq 0.05$ was considered to be statistically significant.

\section{Acknowledgments}

This study was supported in part by the Netherlands Heart Foundation (grant NHS 2002B157). We would like to thank Anneke Geurts-Moespot, Miriam Kooijman, Bas Schouwenberg, Oscar Lemmers, Huub Straatman, Rebecca Paulus, Jan Groothuis, Bregina Kersten, and Jos Evers for their excellent statistical, technical, and/or practical assistance and Eelco de Koning for critical reading of the manuscript.

\section{References}

Adams V, Lenk K, Linke A, Lenz D, Erbs S, Sandri M, Tarnok A, Gielen S, Emmrich F, Schuler G, Hambrecht R (2004) Increase of circulating endothelial progenitor cells in patients with coronary artery disease after exercise-induced ischemia. Arterioscler. Thromb. Vasc. Biol. 24, 684-690.

Asahara T, Murohara T, Sullivan A, Silver $M$, van der Zee R, Li T, Witzenbichler B, Schatteman G, Isner JM (1997) Isolation of putative progenitor endothelial cells for angiogenesis. Science 275, 964-967.

Asahara T, Takahashi T, Masuda H, Kalka C, Chen D, Iwaguro H, Inai Y, Silver M, Isner JM (1999) VEGF contributes to postnatal neovascularization by mobilizing bone marrow-derived endothelial progenitor cells. EMBO J. 18, 3964-3972.
Celermajer DS, Sorensen KE, Spiegelhalter DJ, Georgakopoulos D, Robinson J, Deanfield JE (1994) Aging is associated with endothelial dysfunction in healthy men years before the age-related decline in women. J. Am. Coll. Cardiol. 24, 471-476.

Chen MC, Yip HK, Chen CJ, Yang CH, Wu CJ, Cheng $\mathrm{Cl}$, Chen $\mathrm{YH}$, Chai HT, Lee CP, Chang HW (2006) No age-related change in circulating endothelial progenitor cells in healthy subjects. Int. Heart J. 47, 95-105.

DeSouza CA, Shapiro LF, Clevenger CM, Dinenno FA, Monahan KD, Tanaka H, Seals DR (2000) Regular aerobic exercise prevents and restores age-related declines in endothelium-dependent vasodilation in healthy men. Circulation 102, 1351-1357.

Eskurza I, Monahan KD, Robinson JA, Seals DR (2004) Effect of acute and chronic ascorbic acid on flow-mediated dilatation with sedentary and physically active human ageing. J. Physiol. 556, 315-324.

Franzoni F, Plantinga Y, Femia FR, Bartolomucci F, Gaudio C, Regoli F, Carpi A, Santoro G, Galetta F (2004) Plasma antioxidant activity and cutaneous microvascular endothelial function in athletes and sedentary controls. Biomed. Pharmacother. 58, 432-436.

Gavin TP, Robinson CB, Yeager RC, England JA, Nifong LW, Hickner RC (2004) Angiogenic growth factor response to acute systemic exercise in human skeletal muscle. J. Appl. Physiol. 96, 19-24.

Green DJ, Walsh JH, Maiorana A, Burke V, Taylor RR, O'Driscoll JG (2004) Comparison of resistance and conduit vessel nitric oxide-mediated vascular function in vivo: effects of exercise training. J. Appl. Physiol. 97, 749-755.

Heiss C, Keymel S, Niesler U, Ziemann J, Kelm M, Kalka C (2005) Impaired progenitor cell activity in age-related endothelial dysfunction. J. Am. Coll. Cardiol. 45, 1441-1448.

Hill JM, Zalos G, Halcox JP, Schenke WH, Waclawiw MA, Quyyumi AA, Finkel T (2003) Circulating endothelial progenitor cells, vascular function, and cardiovascular risk. N. Engl. J. Med. 348, 593-600.

Ishizawa K, Kubo H, Yamada M, Kobayashi S, Suzuki T, Mizuno S, Nakamura T, Sasaki H (2004) Hepatocyte growth factor induces angiogenesis in injured lungs through mobilizing endothelial progenitor cells. Biochem. Biophys. Res. Commun. 324, 276-280.

Kraus RM, Stallings HW 3rd, Yeager RC, Gavin TP (2004) Circulating plasma VEGF response to exercise in sedentary and endurance-trained men. J. Appl. Physiol. 96, 1445-1450.

Laufs U, Urhausen A, Werner N, Scharhag J, Heitz A, Kissner G, Bohm M, Kindermann W, Nickenig G (2005) Running exercise of different duration and intensity: effect on endothelial progenitor cells in healthy subjects. Eur. J. Cardiovasc. Prev. Rehabil. 12, 407-414.

Laufs U, Werner N, Link A, Endres M, Wassmann S, Jurgens K, Miche E, Bohm M, Nickenig G (2004) Physical training increases endothelial progenitor cells, inhibits neointima formation, and enhances angiogenesis. Circulation 109, 220-226.

Miyachi M, Kawano H, Sugawara J, Takahashi K, Hayashi K, Yamazaki K, Tabata I, Tanaka H (2004) Unfavorable effects of resistance training on central arterial compliance: a randomized intervention study. Circulation 110, 2858-2863.

Monahan KD, Tanaka H, Dinenno FA, Seals DR (2001) Central arterial compliance is associated with age- and habitual exercise-related differences in cardiovagal baroreflex sensitivity. Circulation 104, 16271632.

Morici G, Zangla D, Santoro A, Pelosi E, Petrucci E, Gioia M, Bonanno A, Profita M, Bellia V, Testa U, Bonsignore MR (2005) Supramaximal exercise mobilizes haematopoietic progenitors and reticulocytes in athletes. Am. J. Physiol. Regul. Integr. Comp. Physiol. 289, R1496-R1503.

Orlic D, Kajstura J, Chimenti S, Jakoniuk I, Anderson SM, Li B, Pickel J, McKay R, Nadal-Ginard B, Bodine DM, Leri A, Anversa P (2001) Bone marrow cells regenerate infarcted myocardium. Nature 410, 701705. 
Ozuyaman B, Ebner P, Niesler U, Ziemann J, Kleinbongard P, Jax T, Godecke A, Kelm M, Kalka C (2005) Nitric oxide differentially regulates proliferation and mobilization of endothelial progenitor cells but not of hematopoietic stem cells. Thromb. Haemost. 94, 770-772.

Paffenbarger RS Jr, Hyde RT, Wing AL, Hsieh CC (1986) Physical activity, all-cause mortality, and longevity of college alumni. N. Engl. J. Med. 314, 605-613.

Palange P, Testa U, Huertas A, Calabro L, Antonucci R, Petrucci E, Pelosi E, Pasquini L, Satta A, Morici G, Vignola MA, Bonsignore MR (2006) Circulating haemopoietic and endothelial progenitor cells are decreased in COPD. Eur. Respir. J. 27, 529-541.

Rauscher FM, Goldschmidt-Clermont PJ, Davis BH, Wang T, Gregg D, Ramaswami P, Pippen AM, Annex BH, Dong C, Taylor DA (2003) Aging, progenitor cell exhaustion, and atherosclerosis. Circulation 108, 457-463.

Rehman J, Li J, Parvathaneni L, Karlsson G, Panchal VR, Temm CJ, Mahenthiran J, March KL (2004) Exercise acutely increases circulating endothelial progenitor cells and monocyte-/macrophage-derived angiogenic cells. J. Am. Coll. Cardiol. 43, 2314-2318.

Ryan NA, Zwetsloot KA, Westerkamp LM, Hickner RC, Pofahl WE, Gavin TP (2006) Lower skeletal muscle capillarization and VEGF expression in aged vs. young men. J. Appl. Physiol. 100, 178-185.

Sandri M, Adams V, Gielen S, Linke A, Lenk K, Krankel N, Lenz D, Erbs S, Scheinert D, Mohr FW, Schuler G, Hambrecht R (2005) Effects of exercise and ischemia on mobilization and functional activation of blood-derived progenitor cells in patients with ischemic syndromes: results of 3 randomized studies. Circulation 111, 3391-3399.

Schmidt-Lucke C, Rossig L, Fichtlscherer S, Vasa M, Britten M, Kamper U, Dimmeler S, Zeiher AM (2005) Reduced number of circulating endothelial progenitor cells predicts future cardiovascular events: proof of concept for the clinical importance of endogenous vascular repair. Circulation 111, 2981-2987.

Shaffer RG, Greene S, Arshi A, Supple G, Bantly A, Moore JS, Mohler ER 3rd (2006) Flow cytometric measurement of circulating endothelial cells: the effect of age and peripheral arterial disease on baseline levels of mature and progenitor populations. Cytometry B. Clin. Cytom. 70, $56-62$.

Shintani S, Murohara T, Ikeda H, Ueno T, Honma T, Katoh A, Sasaki K, Shimada T, Oike Y, Imaizumi T (2001) Mobilization of endothelial progenitor cells in patients with acute myocardial infarction. Circulation 103, 2776-2779.

Span PN, Grebenchtchikov N, Geurts-Moespot J, Westphal JR, Lucassen AM, Sweep CG (2000) EORTC receptor and biomarker study group report: a sandwich enzyme-linked immunosorbent assay for vascular endothelial growth factor in blood and tumor tissue extracts. Int. J. Biol. Markers 15, 184-191.

Steiner S, Niessner A, Ziegler S, Richter B, Seidinger D, Pleiner J, Penka M, Wolzt M, Huber K, Wojta J, Minar E, Kopp CW (2005) Endurance training increases the number of endothelial progenitor cells in patients with cardiovascular risk and coronary artery disease. Atherosclerosis 181, 305-310.

Taddei S, Galetta F, Virdis A, Ghiadoni L, Salvetti G, Franzoni F, Giusti C, Salvetti A (2000) Physical activity prevents age-related impairment in nitric oxide availability in elderly athletes. Circulation 101, 2896-2901.

Tanaka H, Dinenno FA, Monahan KD, Clevenger CM, DeSouza CA, Seals DR (2000) Aging, habitual exercise, and dynamic arterial compliance. Circulation 102, 1270-1275.

Tanaka H, Seals DR, Monahan KD, Clevenger CM, DeSouza CA, Dinenno FA (2002) Regular aerobic exercise and the age-related increase in carotid artery intima-media thickness in healthy men. J. Appl. Physiol. 92, 1458-1464.

Urbich C, Dimmeler S (2004) Endothelial progenitor cells: characterization and role in vascular biology. Circ. Res. 95, 343-353.

Vasa M, Fichtlscherer S, Aicher A, Adler K, Urbich C, Martin H, Zeiher AM, Dimmeler S (2001) Number and migratory activity of circulating endothelial progenitor cells inversely correlate with risk factors for coronary artery disease. Circ. Res. 89, E1-E7.

Werner N, Kosiol S, Schiegl T, Ahlers P, Walenta K, Link A, Bohm M, Nickenig $G$ (2005) Circulating endothelial progenitor cells and cardiovascular outcomes. N. Engl. J. Med. 353, 999-1007. 